\title{
sciendo
}

Current Issues in Pharmacy and Medical Sciences

Formerly ANNALES UNIVERSITATIS MARIAE CURIE-SKLODOWSKA, SECTIO DDD, PHARMACIA

journal homepage: http://www.curipms.umlub.pl/

\section{Effect of tirapazamine on selected kidney parameters in rats treated with cisplatin}

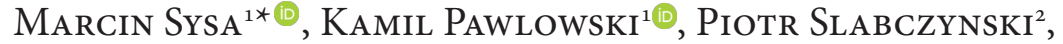 \\ FranCISZEK BURDAN $^{2}{ }^{\oplus}$, JARoslaW DUdKa ${ }^{1 \oplus}$
}

${ }^{1}$ Chair and Department of Toxicology, Medical University of Lublin, 8b Jaczewskiego, 20-090 Lublin, Poland

${ }^{2}$ Human Anatomy Department, Medical University of Lublin, Poland

\section{ARTICLE INFO}

Received 19 March 2018

Accepted 28 May 2018

\section{Keywords:}

Cisplatin,

Tirapazamine,

Nephrotoxicity.

\begin{abstract}
Hypoxic cancer cells are more aggressive and responsible for more efficient metastasis and recurrence. It seems worth-while, hence, to supplement current cytostatic drugs therapy (i.e. cisplatin) with hypoxia cytotoxic agents (i.e. tirapazamine), the toxicity of which is activated by hypoxia. Cisplatin and tirapazamine can change a redox equilibrium and consequently lead to changes in cell metabolism, fibrosis and apoptosis. The aim of this study was to evaluate the cisplatin/tirapazamine toxicological synergism. In doing so we tested selected kidney oxidative stress parameters, as well as nephrotoxicity markers, in plasma and urine. Once a week for 6 weeks, rats received intraperitoneally two doses of tirapazamine ( $5 \mathrm{or} 10 \mathrm{mg} / \mathrm{kg} \mathrm{bw}), 2$ hours before cisplatin $(2 \mathrm{mg} / \mathrm{kg} \mathrm{bw})$ was applied. Our results show that Tirapazamine (TP) had no significant adverse effect on the redox balance, oxidative stress and kidney function in rats receiving cisplatin (CP). However, $\mathrm{TP}$ significantly increased protein concentration in the kidneys of rats. In all tested groups, a significant decrease in NADH concentration in kidneys was recorded, which could indicate disorder in the cell metabolism. TP also was found to have prevented bacterial infection caused by CP. In summary, there was no nephrotoxic synergy of TP with CP at an unacceptable level.
\end{abstract}

\section{INTRODUCTION}

The presence of cancer cells resistant to standard therapeutic methods is a very common problem in anticancer treatment. Many solid tumors contain hypoxia cells, resistant to ionizing radiation as well as chemotherapeutics [1]. To overcome such resistance, a new class of bioreductive drugs was proposed. The most popular is tirapazamine (TP) [2]. As TP is active against only hypoxic cells, it can be used in co-therapy with standard anticancer drugs [3]. A very good example of such combination therapy is the administration of bioreductive drugs, such as tirapazamine, together with other agents such as doxorubicin, cyclophosphamide, 5-fluoruracyl or cisplatin [3]. In the cell, whether normal or cancerous, TP is reduced through various NADPH and NADH reductases to TP radical (TP*-). At normoxia, TP*- is oxidized by oxygen, but under hypoxia conditions such oxidation is strongly limited and TP*-damages the DNA $[4,5]$. There are multiple literature records regarding TP cytotoxicity on cancer cells, but there is limited data on

\footnotetext{
* Corresponding author

e-mail: benefita1985@yahoo.pl
}

the toxic effect of TP on healthy cells [6]. Due to the high dependency of TP on cellular oxygen levels that limits its efficiency in normal cells, tirapazamine is usually used in combination with other therapeutic strategies - radiotherapy or chemotherapeutic drugs such as cisplatin [7].

Cisplatin (cis-diamminedichloroplatinum, CP) is a platinum inorganic complex that has been used in cancer therapy for over 40 years. Its high efficiency in treating various human cancer cells, including bladder, head and neck, lung, ovarian and testicular cancer, makes CP a very popular chemotherapeutic drug $[8,9,10]$. Upon entering the cancer cell, cisplatin undergoes activation. Herein, chloride particles are replaced with water, and the newly formed particle is highly electrophilic and interacts with DNA. This results in crosslinking, impairment of DNA repair mechanisms and eventually promotion of apoptosis [11]. Although its has a wide spectrum of use, CP is reported to contribute to various side effects. Most problematic is nephrotoxicity [12], as the kidney exhibits the highest platinum accumulation rate. Hence, it is a critical organ for cisplatin-based therapy [13]. 
Previous studies have shown that combined therapy of cisplatin and tirapazamine is very effective against solid tumors such as NSCLC or cervix cancer [14]. Still, although Phase I and II clinical trials have yielded promising results, little is known with regard to the modulation of potential side effects of this combined therapy [15]. The lack of information on this issue was the inspiration to undertake the on-going study on the role of tirapazamine in cisplatininduced nephrotoxicity.

\section{MATERIALS AND METHODS}

ANIMALS: The experiment was carried out on adult male Wistar rats (Farm of Laboratory Rats, Brwinow, Poland) weighing 150-180 g. All animals were kept under normal laboratory conditions $\left(22^{\circ} \mathrm{C}, 12 \mathrm{~h}\right.$ day/night cycle $)$ with free access to water and food. The rats were adapted to the laboratory conditions for 7 days before the start of the experiment. All procedures were carried out in accordance with National Institute of Health Guidelines for the Care and Use of Laboratory Animals as well as European Community Council Directive of 24 November 1986 for Care and Use of Laboratory Animals and approved by the Local Ethics Committee.

The animals were placed into six groups, as follows: I - Control group (saline); II - Cisplatin 2mg/kg (CP); III - Tirapazamine $5 \mathrm{mg} / \mathrm{kg}$ (5TP); IV - Tirapazamine $10 \mathrm{mg} / \mathrm{kg}$ (10TP); V - Tirapazamine $5 \mathrm{mg} / \mathrm{kg}+$ Cisplatin $2 \mathrm{mg} / \mathrm{kg}$ $(5 \mathrm{TP}+\mathrm{CP}) ; \mathrm{VI}-$ Tirapazamine $10 \mathrm{mg} / \mathrm{kg}+$ Cisplatin $2 \mathrm{mg} / \mathrm{kg}$ $(10 \mathrm{TP}+\mathrm{CP})$. The solutions of tirapazamine (ADVANCED TECH. \& IND. CO., LTD., China) and Cisplatin (Cefarm, Lublin, Poland) were prepared ex tempore before every administration in saline $(0.9 \% \mathrm{NaCl})$. TP was given i.p. in either $5 \mathrm{mg} / \mathrm{kg}$ or $10 \mathrm{mg} / \mathrm{kg}$ two hours prior to CP i.p. administration $(2 \mathrm{mg} / \mathrm{kg})$, All drugs were administered six times, in weekly intervals. All biological materials for further biochemical tests were collected one week after last dosage.

\section{BIOCHEMICAL PARAMETERS}

Blood for biochemical analysis was collected from the left ventricle into anticoagulant coated probes, centrifuged and the plasma was collected and stored at $4^{\circ} \mathrm{C}$. In order to examine kidney parameters, samples of this organ were collected and stored at $-75^{\circ} \mathrm{C}$. On the day of analysis, the kidney samples were homogenized in $20 \mathrm{mM}$ phosphate buffer $(\mathrm{pH}=7.4)$ using a homogenizer equipped with a Teflon pestle. The homogenate was then centrifuged and the supernatant collected for further analysis. Urine was collected on the last day of the experiment from metabolic cages (SIMAX, Czech Republic), each designated for the individual rat in each group. All urine parameters were tested using specific urine test strips for automated urinalysis (Roche Diagnostics, USA). The following parameters were determined via Power Wave XS spectrophotometric plate reader: GSH/GSSG (Calbiochem, USA) ratio and total glutathione, total protein (Cormay, Poland), lipid peroxidation products (MDA+4HAE; Biotech LPO-586TM - OxisResearchTM, USA) concentration. For determining NADH and NADPH
(Bio Vision, USA), a Victor III Fluorescence Reader (Perkin Elmer, USA) was used.

STATISTICS: All obtained data was processed using STATISTICA software. The statistical significance of differences between the control group and the tested groups was determined with either Manna-Whitney U test or t-Student test. The differences were classified as significant if $\mathrm{p}<0.05$. To compare more than two groups, one-way analysis of ANOVA variance and post-hoc tests of multiple comparisons (HSD Tukey or Dunnett's test) were used. A 5\% error of inference and associated significance level $\mathrm{p}<0.05$ indicating the presence of statistically significant differences or dependencies was accepted.

\section{RESULTS}

Our experiment revealed that the GSH/GSSG ratio was elevated in all the tested groups compared to the control group, except for the group where lower (5TP) tirapazamine concentration was administered (Table 1). Moreover, statistically significant changes in total glutathione concentration in comparison with the control group were exhibited only in this particular group (5TP) (Table 2). Herein, concentration of this parameter was decreased by almost $12 \%$ in comparison with the control group. There was no observed impact of tirapazamine on either GSH/GSSG ratio or total glutathione concentration as compared to the groups receiving only cisplatin. The study revealed elevated concentration of lipid peroxidation products (MDA+4HAE) only in the group of animals where CP and $5 \mathrm{mg} / \mathrm{kg}$ TP was administered together (Table 3), as compared to the control group. Such statistically significant effect was not observed for the animals that had received either 10TP $+\mathrm{CP}$ or CP alone. No statistically significant differences in rat's kidney NADPH concentration in any of tested groups were observed (Table 4, whereas rats in all tested groups had significantly lowered NADH concentration in comparison with the control group (Table 5). Furthermore, combined administration of TP and CP (both $5 \mathrm{mg} / \mathrm{kg}$ and $10 \mathrm{mg} / \mathrm{kg}$ TP groups) resulted in increased total protein concentration as compared to the control group (Table 6). Urinalysis determinations revealed that the $\mathrm{pH}$ level in $5 \mathrm{TP}+\mathrm{CP}$ group was under 7.0 - which indicated acidity, whereas $\mathrm{pH}$ in other groups remained at the same level as the control group (Table 7). The specific gravity of urine remained unaffected (Table 8). Urine analysis revealed no statistically significant changes in the concentration of glucose, urobilinogen, bilirubin, nitrates or erythrocytes in any of the tested groups as compared to the control group (Table 9). Still, significant changes in protein concentration were observed for both $5 \mathrm{TP}+\mathrm{CP}$ and $10 \mathrm{TP}+\mathrm{CP}$ versus $\mathrm{CP}$ alone group, clearly showing that the TP addition in these groups resulted in increased protein concentration. Additionally, urinalysis demonstrated the protective activity of TP in case of preventing potential bacterial infection caused by CP.

To sum up, the analysis of aforementioned parameters lead to a conclusion that only in the case of protein concentration and bacterial flora, we could observe statistical significance between $\mathrm{CP}$ alone group and groups receiving TP and CP together. 
Table 1. GSH/GSSG ratio in the rat kidney

\begin{tabular}{|l|c|c|c|c|c|c|c|}
\hline & N & M & Me & Min & Max & SD & $p$ \\
\hline Control & 5 & 5.58 & 3.73 & 3.24 & 11.96 & 3.682 & \\
\hline CP & 5 & 10.89 & 10.71 & 10.13 & 11.81 & 0.684 & $\mathbf{0 . 0 1 3 2 1 8}$ \\
\hline 5TP & 5 & 9.00 & 9.44 & 7.47 & 10.23 & 1.117 & 0.081580 \\
\hline $10 T P$ & 5 & 10.96 & 10.13 & 9.58 & 13.66 & 1.740 & $\mathbf{0 . 0 1 8 3 2 0}$ \\
\hline 5TP+CP & 5 & 10.64 & 10.52 & 9.84 & 11.35 & 0.693 & $\mathbf{0 . 0 1 6 5 6 1}$ \\
\hline 10TP+CP & 4 & 11.48 & 11.41 & 10.71 & 12.38 & 0.762 & $\mathbf{0 . 0 1 7 0 6 2}$ \\
\hline
\end{tabular}

Table 2. Total glutathione concentration in the rat kidney [nmol/g]

\begin{tabular}{|l|c|c|c|c|c|c|c|}
\hline & N & M & Me & Min & Max & SD & $p$ \\
\hline Control & 5 & 272.77 & 271.53 & 265.31 & 283.95 & 8.101 & \\
\hline CP & 5 & 271.53 & 271.53 & 259.10 & 290.17 & 11.624 & 0.849409 \\
\hline 5TP & 5 & 239.22 & 240.46 & 228.03 & 246.67 & 6.806 & $\mathbf{0 . 0 0 0 1 0 3}$ \\
\hline $10 T P$ & 5 & 270.28 & 265.31 & 259.10 & 302.59 & 18.327 & 0.788543 \\
\hline 5TP+CP & 5 & 265.31 & 271.53 & 252.89 & 271.53 & 8.787 & 0.200535 \\
\hline $10 T P+C P$ & 4 & 278.40 & 274.63 & 271.53 & 292.80 & 10.039 & 0.381301 \\
\hline
\end{tabular}

Table 3. Concentration of lipid peroxidation products (MDA+ 4HAE) in the rat kidney [nmol/g]

\begin{tabular}{|l|c|c|c|c|c|c|c|}
\hline & N & M & Me & Min & Max & SD & $p$ \\
\hline Control & 5 & 29.64 & 29.78 & 25.97 & 33.24 & 2.644 & \\
\hline CP & 5 & 27.22 & 27.35 & 22.51 & 32.89 & 4.195 & 0.250593 \\
\hline 5TP & 5 & 29.16 & 28.39 & 28.05 & 31.51 & 1.497 & 0.530870 \\
\hline $10 T P$ & 5 & 31.79 & 31.51 & 28.74 & 34.28 & 2.223 & 0.143673 \\
\hline 5TP+CP & 5 & 36.15 & 35.32 & 31.16 & 43.63 & 4.656 & $\mathbf{0 . 0 1 6 2 9 4}$ \\
\hline 10TP+CP & 4 & 32.03 & 32.89 & 27.35 & 34.97 & 3.375 & 0.220672 \\
\hline
\end{tabular}

Table 4. Concentration of NADPH in the rat kidney [\% of mean of control]

\begin{tabular}{|l|c|c|c|c|c|c|c|}
\hline & N & M & Me & Min & Max & SD & $p$ \\
\hline Control & 5 & 100.00 & 88.12 & 82.87 & 145.02 & 16.518 & \\
\hline CP & 5 & 91.72 & 93.69 & 81.02 & 97.40 & 4.053 & 0.509183 \\
\hline 5TP & 5 & 99.87 & 96.78 & 92.15 & 117.19 & 6.430 & 0.992348 \\
\hline $10 T P$ & 5 & 93.08 & 92.15 & 88.44 & 99.87 & 3.122 & 0.575156 \\
\hline 5TP+CP & 5 & 84.10 & 82.26 & 77.62 & 96.17 & 4.596 & 0.225134 \\
\hline $10 T P+C P$ & 4 & 89.23 & 89.09 & 86.89 & 91.85 & 1.377 & 0.442616 \\
\hline
\end{tabular}

Table 5. Concentration of NADH in the rat kidney [\% of mean of control]

\begin{tabular}{|l|c|c|c|c|c|c|c|}
\hline & N & M & Me & Min & Max & SD & $p$ \\
\hline Control & 5 & 100.00 & 94.29 & 90.83 & 129.02 & 22.612 & \\
\hline CP & 5 & 32.10 & 27.23 & 21.36 & 61.06 & 22.858 & $\mathbf{0 . 0 0 0 1 8 0}$ \\
\hline $5 T P$ & 5 & 32.82 & 36.55 & 12.05 & 42.41 & 16.478 & $\mathbf{0 . 0 0 0 0 7 3}$ \\
\hline $10 T P$ & 5 & 33.55 & 32.04 & 11.59 & 59.56 & 24.274 & $\mathbf{0 . 0 0 0 2 5 8}$ \\
\hline 5TP+CP & 5 & 28.88 & 26.93 & 16.25 & 42.72 & 14.741 & $\mathbf{0 . 0 0 0 0 3 8}$ \\
\hline $10 T P+$ CP & 4 & 24.73 & 24.34 & 16.10 & 34.14 & 10.959 & $\mathbf{0 . 0 0 0 0 6 7}$ \\
\hline
\end{tabular}

Table 6. Total protein concentration in the rat kidney $[\mathrm{mg} / \mathrm{g}$ of tissue]

\begin{tabular}{|l|c|c|c|c|c|c|c|}
\hline & N & M & Me & Min & Max & SD & $p$ \\
\hline Control & 5 & 81.03 & 83.65 & 68.52 & 89.20 & 8.193 & \\
\hline CP & 5 & 76.19 & 76.59 & 73.06 & 80.63 & 3.057 & 0.250628 \\
\hline 5 TP & 5 & 85.07 & 82.64 & 69.53 & 104.84 & 12.831 & 0.569644 \\
\hline $10 T P$ & 4 & 83.23 & 82.29 & 81.13 & 87.18 & 2.858 & 0.628436 \\
\hline 5TP+CP & 5 & 101.51 & 101.82 & 94.25 & 109.38 & 5.602 & $\mathbf{0 . 0 0 1 7 2 2}$ \\
\hline 10TP+CP & 5 & 97.38 & 93.24 & 89.71 & 114.93 & 10.200 & $\mathbf{0 . 0 2 3 4 1 6}$ \\
\hline
\end{tabular}

Table 7. $\mathrm{pH}$ level of urine

\begin{tabular}{|l|c|c|c|c|c|c|c|}
\hline & $N$ & $M$ & Me & Min & Max & SD & $p$ \\
\hline Control & 8 & 7.00 & 7.00 & 6.00 & 8.00 & 0.535 & \\
\hline CP & 7 & 6.43 & 6.00 & 6.00 & 7.00 & 0.535 & 0.059401 \\
\hline 5TP & 5 & 7.60 & 7.00 & 7.00 & 9.00 & 0.894 & 0.154068 \\
\hline $10 T P$ & 5 & 7.80 & 8.00 & 7.00 & 9.00 & 0.837 & 0.057138 \\
\hline 5TP+CP & 5 & 6.20 & 6.00 & 6.00 & 6.50 & 0.274 & $\mathbf{0 . 0 1 0 6 8 1}$ \\
\hline $10 T P+C P$ & 7 & 6.64 & 6.50 & 6.00 & 8.00 & 0.748 & 0.301936 \\
\hline
\end{tabular}

Table 8. Specific gravity of urine $\left[\mathrm{g} / \mathrm{cm}^{3}\right]$

\begin{tabular}{|l|c|c|c|c|c|c|c|}
\hline & $\mathrm{N}$ & $\mathrm{M}$ & $\mathrm{Me}$ & Min & Max & SD & $\mathrm{p}$ \\
\hline Control & 8 & 1.016 & 1.015 & 1.010 & 1.025 & 0.0050 & \\
\hline CP & 8 & 1.018 & 1.018 & 1.015 & 1.025 & 0.0037 & 0.272934 \\
\hline $5 T P$ & 5 & 1.012 & 1.010 & 1.010 & 1.015 & 0.0027 & 0.165810 \\
\hline $10 T P$ & 5 & 1.013 & 1.015 & 1.010 & 1.015 & 0.0027 & 0.305459 \\
\hline 5TP+CP & 5 & 1.017 & 1.015 & 1.015 & 1.020 & 0.0027 & 0.584721 \\
\hline $10 T P+C P$ & 8 & 1.020 & 1.020 & 1.015 & 1.025 & 0.0046 & 0.089431 \\
\hline
\end{tabular}

Table 9. Frequency of occurrence of changes $(\mathrm{x} / \mathrm{N})$ for selected parameters in urine: protein (PRO), glucose (GLU), urobilinogen (UBG), bilirubin (BR), nitrite (NT), erythrocyte (ER), bacterial flora (BF)

\begin{tabular}{|l|c|c|c|c|c|c|c|}
\hline & PRO & GLU & UBG & BR & NT & ER/ $\mu \mathrm{l}$ & $\mathrm{BF}$ \\
\hline Control & $8 / 8(++)$ & $0 / 8$ & $0 / 8$ & $0 / 8$ & $0 / 8$ & $2 / 8$ & $1 / 8$ \\
\hline CP & $7 / 8(+)$ & $0 / 8$ & $0 / 8$ & $0 / 9$ & $0 / 8$ & $0 / 8$ & $7 / 8$ \\
\hline $5 T P$ & $4 / 5(+)$ & $0 / 5$ & $0 / 5$ & $0 / 5$ & $0 / 5$ & $0 / 5$ & $0 / 5$ \\
\hline $10 T P$ & $2 / 5(+)$ & $0 / 8$ & $0 / 5$ & $0 / 5$ & $0 / 5$ & $0 / 5$ & $0 / 5$ \\
\hline $5 T P+C P$ & $4 / 5(++)$ & $0 / 5$ & $0 / 5$ & $0 / 5$ & $0 / 5$ & $0 / 5$ & $0 / 5$ \\
\hline $10 T P+C P$ & $3 / 7(+)$ & $0 / 7$ & $0 / 7$ & $0 / 7$ & $0 / 7$ & $0 / 7$ & $0 / 7$ \\
\hline
\end{tabular}

Semi-quantitative assessment: mean value in group $<25 \mathrm{mg} / \mathrm{dl}=+$;

$>50 \mathrm{mg} / \mathrm{dl}=++$

\section{DISCUSSION}

The most common side effect of cisplatin therapy is nephrotoxicity. This is connected with the relatively high $\mathrm{CP}$ accumulation in the kidney, as well as with the role of this organ in platinum compound elimination. Detailed study of available literature regarding common mechanisms of action for TP and CP has helped to pinpoint redox imbalance as being this mechanism. Therefore, it was important to determine if both tested drugs exacerbated oxidative stress in the kidney.

Previous experiments in both in vitro and in vivo studies have shown that CP stimulates reactive oxygen species (ROS) 
formation [16,17]. Indeed, a Cisplatin dose of $10 \mathrm{mg} / \mathrm{kg}$ leads to impairment of enzymatic antioxidative defense mechanisms (lowered activity of glutathione reductase) and an increase in both ROS formation and lipid peroxidation products concentration [18]. This is further supported by the increase in total protein concentration - indicating drug-induced cytotoxicity.

Compared to CP, the mechanisms of TP action are less known, but there are experiments which indicate that TP is capable of generating ROS. This effect is believed to be connected with the NADPH-dependent process of one and two-electron reduction of TP to its more reactive form (TP*-) [19]. Taking into consideration that both CP and TP could generate ROS, as well as the role of oxidative stress in cisplatin-dependent nephrotoxicity, it could be assumed that combined administration of these drugs might further enhance the cytotoxic effect on the kidney of rats. However, our study showed that the addition of TP to CP had no influence on either kidney oxidative stress or NADPH concentration if compared to $\mathrm{CP}$ alone group. Oxidative stress in kidney, evaluated through lipid peroxidation products (MDA +4HAE) comparison, revealed that only in case of $5 \mathrm{TP}+\mathrm{CP}$ group does such stress occur.

In every group of rats receiving $\mathrm{CP}$, the $\mathrm{GSH} / \mathrm{GSSG}$ ratio was elevated in comparison with the control group. There were, however, no significant differences between $\mathrm{CP}$ and $\mathrm{TP}+\mathrm{CP}$ groups. There were also no important changes in NADPH concentration in either group, while NADH was significantly lowered versus control group, which could indicate disorder in cell metabolism (free fatty acid beta oxidation and/or Krebs cycle inhibition). We didn't, however, observe any synergistic effect for this parameter in case of $\mathrm{TP}+\mathrm{CP}$ groups, thus indicating that TP does not cause oxidative stress nor that is disrupts kidney redox equilibrium in rats receiving cisplatin.

Changes in redox equilibrium in all the groups receiving CP versus control group are seen in the GSH/GSSG ratios. These changes are the result of GSSG reduction alone because the kidney itself does not affect the anabolism/ catabolism of glutathione. This is supported by the fact that in all the tested CP groups, no significant changes in total glutathione concentration could be observed. It could be assumed, therefore, that the increase in GSH/GSSG ratio is a form of biological adaptive response to the cells impaired reduction capability. It should be noted that the material for analysis was collected 7 days after the last administration, therefore, the availability of $\mathrm{CP}$ to form complexes with GSH is significantly lowered. Despite this fact, earlier administration of $\mathrm{CP}$ could stimulate adaptive synthesis of GSH regeneration enzymes and this activity would be sustained even one week after last administration. This helps to explain why the GSH concentration in CP group was relatively higher than its physiological value. In reference to the previous statement regarding the adaptive response of enzymes responsible for GSH regeneration, it could be assumed that not only the activity is increased but also the concentration of NADPH generating enzymes. If the dynamics of generating NADPH and its utilization remain in balance, then even with NAPDH fast rotation in redox processes, its concentration remains the same. This allows us to understand why even though GSH concentration is elevated, the concentration of NADPH remains the same. In our study, no definitive signs of oxidative stress could be observed in the CP only group, while the GSH/GSSG was elevated in comparison with the control group. This indicates an increase in antioxidative defense capability, most likely as a form of adaptation. The incompatibility of our study with literature data regarding renal oxidative stress after $\mathrm{CP}$ administration (Table 6) could be the result of different dose, as well as time between last injection and biological materials collection. Many experiments use a CP dose of $5 \mathrm{mg} / \mathrm{kg}$ up to $15 \mathrm{mg} / \mathrm{kg}$ and their collection time is 72 hours after the last injection [17]. In our experiment, a lower dose $(2 \mathrm{mg} / \mathrm{kg})$ was used and the materials were collected 7 days after last administration.

Urinalysis revealed that tirapazamine had influence upon the rat kidney in which $\mathrm{CP}$ was previously administered only with reference to the protein level. TP increased protein concentration in both $\mathrm{TP}+\mathrm{CP}$ groups versus $\mathrm{CP}$ alone group. However, without histopathological examination, we were unable to determine if the elevation in this particular parameter could result from the ongoing process of fibrosis occurring inside the kidney. Urinalysis also demonstrated the protective activity of TP in preventing potential bacterial infection caused by CP. However, in order to explain this beneficial mechanism of TP, further studies would be required.

Our study did not provide decisive evidence on the potential of tirapazamine to act as protective agent against cisplatin-induced nephrotoxicity. There are still too many questions regarding the effect of tirapazamine on normal cells, as well as potential side effects. Further research, including histological analysis of kidney as well as analysis of apoptosis pathways, would be required to provide satisfactory and conclusive statements regarding the potential of tirapazamine to efficiently decrease cisplatin-induced nephrotoxicity.

\section{ORCID iDs}

Marcin Sysa (Dhttps://orcid.org/0000-0002-7108-5772

Kamil Pawłowski (Dhttps://orcid.org/0000-0002-9646-9959

Franciszek Burdan (Dhttps://orcid.org/0000-0001-5305-8829

Jarosław Dudka Dhttps://orcid.org/0000-0002-9801-9054

\section{CONCLUSIONS}

1. Unexpected increase in GSH/GSSG ratio in the tested group vs control after 7 days post last drug administration probably results from adaptive redox balance mechanism.

2. Analysis of changes in lipid peroxidation and NADPH concentration leads to the conclusions that tirapazamine does not bring about oxidative stress nor does it disrupt redox equilibrium in rats received cisplatin.

3. Decrease in the NADH parameter in all tested groups may result from Krebs cycle inhibition.

4. There were no effects of tirapazamine on kidney GSH/ GSSG ratio, total glutathione, MDA+4HNE, NADPH and NADH concentrations in rats administered with cisplatin.

5. Tirapazamine increases kidney total protein concentration in rats treated with cisplatin. Further studies are necessary to explain if this change is related to fibrosis. 
6. It is interesting to note that tirapazamine protects rats treated with cisplatin from bacterial infections.

7. In summary, there was no nephrotoxic synergy of TP and $\mathrm{CP}$ at an unacceptable level.

\section{REFERENCES}

1. Tredan O, Galmarini CM, Patel K, Tannock IF. Drug resistance and the solid tumor microenvironment. J Natl Cancer Inst. 2007;99: 1441-54.

2. Kyle AH, Minchinton AI. Measurement of delivery and metabolism of tirapazamine to tumour tissue using the multilayered cell culture model. Cancer Chemother Pharmacol. 1999;43:213-20.

3. Rischin D, Peters L, Fisher R. Tirapazamine, cisplatin, and radiation versus fluorouracil, cisplatin, and radiation in patients with locally advanced head and neck cancer: a randomized phase II trial of the Trans-Tasman Radiation Oncology Group (TROG 98.02). J Clin Oncol. 2005;23:79-87.

4. Hicks KO, Myint H, Patterson AV, Pruijn FB, Siim BG, Patel K, Wilson WR. Oxygen dependence and extravascular transport of hypoxia-activated prodrugs: Comparison of the dinitrobenzamide mustard PR-104A and tirapazamine. Int J Radiat Oncol Biol Phys. 2007;69:560-71.

5. Marcu L, Olver I. Tirapazamine: from bench to clinical trials. Curr Clin Pharmacol. 2006;1:71-9.

6. Rischin D, Peters LJ, O'Sullivan B. Tirapazamine, cisplatin, and radiation versus cisplatin and radiation for advanced squamous cell carcinoma of the head and neck (TROG 02.02, HeadSTART): a phase III trial of the Trans-Tasman Radiation Oncology Group. J Clin Oncol. 2010;28:2989-95.

7. Di Silvestro PA, Ali S, Craighead PS. Phase III randomized trial of weekly cisplatin and irradiation versus cisplatin and tirapazamine and irradiation in stages IB2, IIA, IIB, IIIB, and IVA cervical carcinoma limited to the pelvis: a Gynecologic Oncology Group study. J Clin Oncol. 2014;32:458-64.
8. Chen HHW, Kuo MT. Role of Glutathione in the Regulation of Cisplatin Resistance in Cancer Chemotherapy. Metal-Based Drugs. 2010;2010:430939.

9. Ozkan TA, Aydin U, Ay D, Cebeci IOO. Cisplatin and bleomycininduced acute peripheral-vascular stenosis in patient with testicular cancer. Urology Annals. 2016;8:483-5.

10. Qian X, Ma C, Hoffmann TK, Kaufmann AM, Albers AE. Taxanecisplatin-fluorouracil as induction chemotherapy for advanced head and neck cancer: a meta-analysis of the 5-year efficacy and safety. SpringerPlus. 2015;4:208.

11. Dasari S, Tchounwou PB. Cisplatin in cancer therapy: molecular mechanisms of action. Eur J Pharmacol. 2014;740:364-78.

12. Miller RP, Tadagavadi RK, Ramesh G, Reeves WB. Mechanisms of Cisplatin Nephrotoxicity. Toxins. 2010;2:2490-518.

13. Arany I, Safirstein RL. Cisplatin nephrotoxicity. Semin. Nephrol. 2003;23:460-4.

14. Guise CP, Mowday AM, Ashoorzadeh A. Bioreductive prodrugs as cancer therapeutics: targeting tumor hypoxia. Chinese Journal of Cancer. 2014;33(2):80-6.

15. Rades D, Seidl D, Janssen S, Strojan P, Karner K, Bajrovic A, Hakim SG. Comparing two lower-dose cisplatin programs for radiochemotherapy of locally advanced head-and-neck cancers. European Archives of Oto-Rhino-Laryngology. 2017;1:1-7.

16. Rabik C, Fishel M, Holleran J, Kasza K, Kelley M, Egorin M, Dolan M. Enhancement of cisplatin cytotoxicity by O6-benzylguanine involves endoplasmic reticulum stress. J. Pharmacol. Exp. Ther. 2008;327:442-52.

17. Santos NA, Catao CS, Martins NM, Curti C, Bianchi ML, Santos AC. Cisplatin-induced nephrotoxicity is associated with oxidative stress, redox state unbalance, impairment of energetic metabolism and apoptosis in rat kidney mitochondria. Arch Toxicol. 2007;81(7): 495-504.

18. Kadikoylu G, Bolaman Z, Demir S, Balkaya M, Akalin N, Enli Y. The effects of desferrioxamine on cisplatin-induced lipid peroxidation and the activities of antioxidant enzymes in rat kidneys. Hum Exp Toxicol. 2004;23:29-34.

19. Delahoussaye YM, Evans JW, Brown JM. Metabolism of tirapazamine by multiple reductases in the nucleus. Biochem Pharmacol. 2001;62(9):1201-9. 